\title{
E-Commerce Enterprise Marketing Management: Realistic Dilemma, Main Incentives and Resolution Strategies
}

\author{
Chenjie Li \\ Shanghai University, Shanghai, China \\ Email:rli@cjcomponent.com
}

How to cite this paper: Li, C. J. (2020). E-Commerce Enterprise Marketing Management: Realistic Dilemma, Main Incentives and Resolution Strategies. Open Journal of Social Sciences, 8, 245-251.

https://doi.org/10.4236/iss.2020.87020

Received: June 28, 2020

Accepted: July 20, 2020

Published: July 23, 2020

Copyright $\odot 2020$ by author(s) and Scientific Research Publishing Inc. This work is licensed under the Creative Commons Attribution International License (CC BY 4.0).

http://creativecommons.org/licenses/by/4.0/

\section{(c) (i) Open Access}

\begin{abstract}
During the period of economic transformation, the competition in the e-commerce industry is becoming increasingly fierce. The implementation of the business strategy of "cost reduction, efficiency increase, and quality improvement" has become an inevitable direction and beneficial exploration for e-commerce companies to implement transformation and development. Currently, e-commerce enterprise marketing has the problems of increasing customer acquisition costs, declining overall efficiency, and low operational efficiency. The main reason for this situation is that e-commerce enterprise marketing management lacks refined management, innovative exploration, and digital operations. Based on this, this article believes that e-commerce companies should restructure the marketing management system, promote the innovation of marketing models, and accelerate the reserve of high-quality talents, so as to truly enhance the appeal, influence, and competitiveness of e-commerce companies.
\end{abstract}

\section{Keywords}

E-Commerce Enterprise, Marketing Management, Practical Dilemma, Main Incentives, Coping Strategies

\section{Introduction}

With the rapid development and widespread popularity of my country's Internet technology, the e-commerce industry has gained leaping development opportunities, and a large number of e-commerce companies have begun to appear. According to the statistics of China News Network, since 2013, China's e-commerce enterprises have shown a rapid development trend. Although the growth 
rate has slowed down in 2016, it has remained at around $40 \%$. In 2017, the growth rate reached a peak $74.95 \%$ (Jin, 2019). However, with the release of the online Internet dividend nearing its end, many e-commerce companies have been forced out of the market competition for the survival of the fittest. The main reason is that their marketing management is difficult to achieve effective results and cannot achieve sustainable development (Fu, 2019).

According to the " 315 E-commerce Big Data" released by Tianyancha in 2020, as of the beginning of 2020, there were nearly 3 million enterprises with "e-commerce and Internet sales" in their national business names or business scope. However, due to various problems in operation, so far, a total of 430,000 home appliance companies in the country have shown cancellation or revocation, accounting for nearly $15 \%$ of the total number of companies. Among them, the main reason for the failure of e-commerce companies is that the marketing situation has not been opened. Unable to maintain (Zhang et al., 2019) from this, we can see that marketing work is very important for e-commerce companies. In view of this, this article focuses on the marketing management of e-commerce companies, starting with the actual marketing dilemmas faced by e-commerce companies, focusing on the causes of e-commerce companies' marketing dilemmas, and on this basis, exploring specific resolution strategies to e-commerce companies Marketing management is beneficial.

\section{The Practical Dilemma of E-Commerce Enterprise Marketing Management}

Analyzing the practical dilemma existing in the marketing management of e-commerce companies is a targeted analysis of the incentives and the basis and premise of solving countermeasures. This article analyzes the current problems of marketing management of e-commerce enterprises from three levels of marketing acquisition, marketing effectiveness, and operating efficiency.

\subsection{The Cost of Acquiring Customers for Marketing Has Increased, and the "Cost Reduction" Effect of E-Commerce Companies Is Generally Poor}

The increase in customer acquisition cost of marketing is the primary problem in the marketing management of e-commerce companies. The direct consequence is that the "cost reduction" effect of e-commerce companies is generally poor. In fact, in the early days of the development of the e-commerce industry, online customer traffic dividends are relatively rich, e-commerce companies' unit online customer acquisition costs are not high, and the e-commerce enterprise's marketing input-output ratio is relatively considerable, which makes Various enterprises established in the early stage of the industry development have obtained leaping development opportunities (Zhang, 2016). However, with the release of the initial traffic bonus in the industry and the increase in online marketing competitors, the online customer acquisition environment of e-commerce 
companies has gradually deteriorated, and the competition for marketing activities, marketing methods and marketing strategies has become increasingly fierce. The online customer acquisition costs continue to increase, which undoubtedly makes it difficult for e-commerce companies to achieve the goal of reducing customer acquisition costs.

\subsection{The Overall Effectiveness of Marketing Has Declined, and the "Efficiency" Goal of E-Commerce Companies Is Difficult to Achieve}

The decline in the overall effectiveness of marketing is a core problem in the marketing management of e-commerce companies, which will make it difficult for e-commerce companies to achieve the goal of "increasing efficiency." (Wang, 2016). For e-commerce companies, online customer acquisition is a very important way of influencing, so the overall effectiveness of marketing depends on two major links: first, the cost of marketing customer acquisition is low and the effect is good; second, In the marketing service, customers can create higher income for enterprises. In the marketing customer acquisition process, as mentioned earlier, due to the increasingly fierce marketing competition, the difficulty of acquiring customers for e-commerce companies is increasing, and cost control is also extremely difficult. In the marketing service segment, e-commerce companies currently lack effective methods, means and tools to increase customer attention, activity and stickiness, which makes it difficult for e-commerce companies to ensure the achievement of revenue-generating goals, while also indirectly improving The cost of e-commerce enterprise customer marketing (Bao, 2017).

\subsection{The Operational Efficiency of Marketing Is Low, and It Is Difficult For E-Commerce Companies to Meet the "Quality Improvement" Requirements}

The low operating efficiency of marketing is one of the main problems in the marketing management of e-commerce enterprises, and its main drawback is that it makes it difficult for e-commerce companies to meet the "quality improvement" requirements. First, many e-commerce companies are unable to achieve precision, efficiency, and intelligence in marketing, which makes the marketing objectives unclear, the operation of all aspects of marketing is inefficient, and the input-output ratio of marketing is very inefficient; second, As it is difficult to improve the efficiency of marketing operations, the secondary marketing effect of e-commerce companies is not significant, which makes it difficult to carry out product marketing and business mining on existing stock customers, thus restricting the sustainable development of e-commerce business (Zhong \& Cao, 2015). Furthermore, due to the insufficient operational efficiency of marketing, the lack of coordination in all aspects of marketing is not conducive to the shaping of the overall marketing image of e-commerce companies, which will inhibit the marketing efficiency of e-commerce companies to a certain extent. 


\section{The Main Reason for the Difficulty of Marketing Management in E-Commerce Enterprises}

Based on the analysis of the practical difficulties in the marketing management of e-commerce companies, the author further analyzes the main reasons for the various marketing difficulties of e-commerce companies from the three aspects of refined marketing management, innovative exploration of marketing models, and digital operation of marketing tools. So as to lay the foundation for targeted exploration of countermeasures to solve the marketing dilemma of e-commerce enterprises (Xie, 2019).

\subsection{Marketing Lacks Refined Management, and the Marketing Management System of E-Commerce Companies Lags Behind}

The lack of refined management in marketing is a common problem for e-commerce companies. The specific performance is as follows: first, e-commerce companies lack scientific planning, marketing activities have no plans, marketing objectives are not clear, and there is insufficient effective analysis of target customers. In addition, the lack of continuity in marketing activities and the lack of rigorous marketing content and tools have made the effectiveness of e-commerce companies' marketing efforts very low. Second, the lack of scientific, objective and timely evaluation of e-commerce companies' marketing efforts has made e-commerce companies Marketing deficiencies are difficult to be discovered, and the targeted marketing vulnerabilities cannot be improved, and the marginal efficiency of marketing is difficult to improve. Third, the marketing division of e-commerce companies is unclear, and there are no clear arrangements for responsibilities, powers, and interests. Matching, which is extremely easy to cause friction in internal management, reduces the effectiveness of marketing activities.

\subsection{Marketing Lacks Innovative Exploration, and the Marketing Model of E-Commerce Companies Lacks Innovation}

Marketing innovation is a necessary quality and effective way for e-commerce enterprises to adapt to the competition in the Internet market. However, from a practical point of view, many e-commerce companies generally lack innovative marketing exploration, which seriously restricts the development space of e-commerce companies. Of course, in order to reduce marketing costs, many e-commerce companies are often reluctant to innovate their marketing models. They like to follow the new marketing models and try to replicate the marketing models of other companies to achieve the innovation of marketing models. However, from the practical experience, this approach is difficult to produce efficiency. On the contrary, due to the significant differences in resource endowments, industry attributes, and comprehensive strength of various enterprises, a simple marketing model imitation not only makes it difficult to improve marketing effectiveness, but also increases the operating costs of enterprises. 


\subsection{Lack of Digital Operation in Marketing, Insufficient Marketing Talent Reserve for E-Commerce Companies}

Essentially, e-commerce companies are Internet companies, so they must have Internet operation thinking, strengthen their own collection, analysis, mining, and prediction of data, and formulate their own management and management countermeasures based on the in-depth grasp of data. In order to form efficient, accurate and sustainable competitiveness. However, at present, many e-commerce companies generally lack this kind of digital operation thinking, and still position themselves as traditional retail enterprises, which makes it difficult to use digital technology and digital tools, and cannot truly achieve precise marketing, efficient marketing, and intelligent marketing. Of course, the important problem that makes it difficult for e-commerce companies to implement digital operations is also due to the lack of comprehensive marketing talents for e-commerce companies, which inhibits the development potential of e-commerce companies.

\section{The Countermeasures to Resolve the Dilemma of E-Commerce Enterprise Marketing}

\subsection{Reconstruct the Marketing Management System and Strengthen the Improvement of the Effectiveness of All Aspects of Marketing}

Restructuring the marketing management system is the basic strategy to solve the dilemma of e-commerce enterprise marketing management, and its core goal is to strengthen the improvement of the effectiveness of all aspects of marketing. First, e-commerce companies must establish a marketing management system, clarify the power, responsibility, and benefits of various marketing positions, and effectively incentivize and restrict marketing positions from the system level to ensure the orderly and efficient marketing work; second, E-commerce companies must strengthen the evaluation of marketing effectiveness, including both marketing reserves and planning links, as well as the marketing process and output links, strengthen marketing evaluation work, and force the reform of the marketing evaluation system to enhance the effectiveness of marketing work; third, E-commerce companies must pay attention to the follow-up maintenance of customers and the development of secondary marketing. They must increase the revenue contribution of individual customers, do a good job in customer life cycle management, and give differentiated marketing strategies to different customers at different stages in order to improve the customer's Marketing effectiveness.

\subsection{Implement Marketing Model Innovation and Actively Try Various Online Marketing Methods}

Implementing marketing model innovation is a key strategy to solve the dilemma of e-commerce enterprise marketing management, and its execution direction is to actively try various online marketing methods. For traditional retail 
enterprises, the e-commerce model is an innovation of business model. Similarly, for e-commerce companies, continuous innovation in marketing models is also very important, otherwise it is difficult to adapt to changes in the market and industry, and ultimately it is difficult to obtain a favorable competitive position. Therefore, e-commerce companies must implement marketing model innovation. On the one hand, adhere to the traditional online marketing model to ensure that the marketing work is carried out smoothly, while actively optimizing the deficiencies of the traditional online marketing model to improve marketing efficiency, such as BD, SEM, SEO and other drainage models to optimize and reduce the cost of drainage; another In terms of aspects, we actively tried new online drainage models, such as short video marketing, webcast, VR marketing, etc., and actively implemented new marketing models and marketing plans around the new Internet format to continuously improve the effectiveness of marketing.

\subsection{Accelerate the Reserve of High-Quality Talents and Comprehensively Promote the Digital Management of Enterprises}

Accelerating high-quality talent reserve is an important supplementary strategy to solve the dilemma of e-commerce enterprise marketing management, and it is necessary to base on the talent reserve to fully promote the enterprise's digital management. First, e-commerce companies must adhere to the high-quality talent reserve model of internal cultivation and external introduction, to ensure that e-commerce companies can build a diversified talent who understands e-commerce business, is proficient in digital technology, and is familiar with marketing strategies. Each operation link of a business enterprise is integrated into digital management, and the operation efficiency of the enterprise is improved through digital operation. At the same time, it is also beneficial to the management to formulate marketing directions and plans; in addition, it is necessary to strengthen the analysis of customer behavior to provide e-commerce enterprises. Lay a solid foundation for marketing work.

\section{Research Conclusion}

All in all, with the rapid increase of e-commerce industry participants and the transformation and development of offline companies, e-commerce companies are facing unprecedented competitive challenges. At the same time, with the continuous release of online customer traffic dividends, the operational efficiency of e-commerce companies must also be improved, otherwise it will be difficult to adapt to the "Red Sea" competition in the e-commerce industry.

Through the investigation of current e-commerce companies, this article finds that the current e-commerce companies have problems such as "general cost reduction" is generally poor, "efficiency" goals are difficult to achieve, and "quality improvement" requirements are difficult to meet. The main causes are e-com- 
merce companies. China's marketing management system lags behind, marketing models lack innovation, and marketing talent reserves are insufficient. Based on the current development trend of e-commerce industry and the characteristics of the exhibition industry, combined with the current basic operation status of e-commerce companies, the author recommends that e-commerce companies should restructure the marketing management system, promote the innovation of marketing models, accelerate the high-quality talent reserve, and focus on strengthening the effectiveness of all aspects of marketing Promotion, actively try various online marketing methods, comprehensively promote the digital management of enterprises, and then improve the accuracy, efficiency, and sustainability of e-commerce enterprise marketing, and ultimately enhance the core competitiveness of e-commerce enterprises.

\section{Conflicts of Interest}

The author declares no conflicts of interest regarding the publication of this paper.

\section{References}

Bao, Y. (2017). Analysis of Corporate Marketing Planning Ideas in the Context of eCommerce. Industry and Technology Forum, 16, 276-277.

$\mathrm{Fu}$, L. (2019). Discussion on the Optimization Strategy of e-Commerce Marketing Management under the Background of Big Data. Modern Marketing (Late Journal), 4, 192.

Jin, S. J. (2019). Discussion on Enterprise Marketing Management under e-Commerce Model. SME Management and Technology (Late Issue), 11, 152-153.

Wang, L. L. (2016). Discussion on the Innovation of Marketing Management Mode of Enterprise e-Commerce. Science and Technology Information, 14, 78+80.

Xie, H. M. (2019). Research on e-Commerce Enterprise Marketing Management Based on Data Mining. Brand Research, 11, 35-36.

Zhang, J. (2016). Research on Marketing Management of Small and Medium-Sized Enterprises in My Country-Based on the Perspective of e-Commerce Development. Modern Marketing (Late Journal), 8, 24.

Zhang, J., Zhou, Y. B., Yu, Q., \& Cao, J. W. (2019) Research on Internet Marketing Management in the Mode of Small and Medium-Sized Enterprises E-Commerce. Information Recording Materials, 20, 222-223.

Zhong, M., \& Cao, Y. P. (2015). Research on Marketing Strategies of Small and Medium-Sized e-Commerce Enterprises in the Era of Big Data. Chinese Market, 49, 16-17. 